ARTÍCULO ORIGINAL

\title{
LA MOTIVACIÓN DENTRO DE LA GESTIÓN ADMINISTRATIVA DE LAS PEQUEÑAS EMPRESAS
}

\section{MOTIVATION WITHIN THE ADMINISTRATIVE MANAGEMENT OF SMALL ENTERPRISES}

\author{
Rafael Bermúdez Tacunga \\ Ingeniero Comercial - Magister en Tributación y Finanzas - Docente Principal de la Universidad Laica Eloy Alfaro de Manabí - Ecua- \\ dor, Email: rafabermudez12@yahoo.com (Autor Corresponsal)
}

[Recibido: 12/09/2016 Aceptado: 26/11/2016]

\begin{abstract}
RESUMEN
La gestión administrativa dentro de las pequeñas empresas, tiene como finalidad principal la maximización de las utilidades que se obtiene de la actividad realizada, requiriendo inevitablemente de altos índices de productividad en sus trabajadores, quienes a su vez mantienen variadas motivaciones derivadas de factores tanto internos como externos que determinan el comportamiento que evidencian dentro del desempeño de sus funciones y actividades cotidianas. De lo expuesto, surge el problema que se analiza en el presente trabajo, mismo que se establece como los aspectos de la gestión administrativa que inciden en la motivación que poseen los trabajadores de las pequeñas empresas para el desempeño de sus labores. En el artículo se presenta una revisión bibliográfica sobre los aspectos motivacionales que tienen las personas y se efectúa una descripción de los factores que motivan a los trabajadores en el desempeño de sus funciones, para lo cual se asumió una encuesta a una muestra de 200 trabajadores de planta y 6 jefes operativos que laboran en 6 pequeñas empresas manufactureras. Los principales resultados que se presentan en el trabajo están relacionados por las motivaciones variadas que tienen los trabajadores en función de los niveles educativos y el entorno laboral que poseen.
\end{abstract}

\section{PALABRAS CLAVE}

Motivación, gestión administrativa, desempeño laboral, pequeñas empresas.

\begin{abstract}
The main purpose of administrative management within small enterprises is the maximization of the profits obtained from the accomplished activity, inevitably requiring high rates of productivity in their workers, who in turn maintain varied motivations derived from both internal and external factors that determine the behavior evidenced within the performance of their functions and daily activities. From the above, the problem analyzed in the present work establishes the aspects of the administrative management that affect the motivation that small companies' workers have for the performance of their work. The article presents a literature review on the motivational aspects that people have and a description of the factors that motivate workers in the performance of their duties. A survey was carried out on a sample of 200 plant workers and 6 operating heads working in 6 small manufacturing companies. The main results that are presented in the work are related by the varied motivations that the workers have according to the educational levels and the work environment that they possess.
\end{abstract}

\section{KEYWORDS}

Motivation, administrative management, job performance, small business.

Como Citar: Bermudez, F. (2017). La motivación dentro de la gestión administrativa de las pequeñas empresas. Quipukamayoc 25(47), 37 - 44. doi: http://dx.doi.org/10.15381/quipu.v25i47.13801

\section{INTRODUCCIÓN}

En un contexto productivo laboral cada vez se vuelve más importante conocer aquellos factores que repercuten de manera evidente en el desempeño o rendimiento laboral de las personas que integran algún tipo de organización empresarial. Se conoce que existen ciertos componentes físicos y sociales que influyen sobre el comportamiento humano y es allí donde la motivación para el desempeño laboral determina la forma en que un individuo percibe su trabajo. Maslow (1970) considera que las personas se encuentran está en un constante estado de motivación, y establece que a medida que se satisface un deseo, surge otro en su lugar; a pesar de que el enfoque de Maslow es demasiado amplio, representa para la adminis- tración de recursos humanos un valioso modelo del comportamiento de las personas, basado en la carencia (o falta de satisfacción de una necesidad específica) y complacencia que lleva al individuo a satisfacer dicha necesidad; lo cual implica que cuando una persona cubre una necesidad de menor nivel, disminuye la importancia que le brinda a la misma y se activa paralelamente el siguiente nivel supe- 
rior; es decir no se requiere que una necesidad se satisfaga en su totalidad antes de que emerja la siguiente. En las sociedades modernas muchos trabajadores ya han satisfecho sus necesidades de orden inferior y están motivados por necesidades psicológicas de orden superior. Sin embargo, esto generalmente sucede en países con economías altamente desarrolladas, que poseen niveles remunerativos elevados y entornos laborales adecuados. En países como el Ecuador, y específicamente en la provincia de Manabí, aún existe mucha gente que trabaja en empresas únicamente para satisfacer necesidades primarias, por efecto de los bajos niveles remunerativos que perciben como retribución del trabajo que realizan dentro de la organización, mismo que, en la mayoría de los casos, no cubre el valor estimado para la canasta básica ecuatoriana.

Es necesario acotar que la remuneración que reciben los trabajadores, no es el único factor que motiva su desempeño laboral, existen otros como el ambiente de trabajo, el trato por sus superiores, la estabilidad laboral, la actitud personal, etc. que igualmente afectan su actividad dentro de las pequeñas empresas y que son parte importante del análisis efectuado y expuesto en el presente informe de investigación.

Lo expresado en los párrafos anteriores es el fundamento para la existencia de una contradicción externa que se manifiesta en la demanda, por parte de las empresas, de un eficiente desempeño laboral de los trabajadores en el ejercicio de sus funciones, $y$ el desempeño que los mismos tienen motivados por elementos intrínsecos y extrínsecos de su organización

En las pequeñas empresas privadas de la provincia de Manabí aún persisten las aplicaciones de métodos adminis- trativos del talento humano basados en criterios empíricos donde no se considera el factor emotivo de los trabajadores como parte importante de su satisfacción laboral y consecuente rendimiento en sus labores cotidianas, lo que indudablemente afecta al alcance de objetivos de las citadas organizaciones (Revista Lideres, 2013).

Al respecto, existen estudios expuestos en tesis doctorales sobre temas similares que se exponen como antecedentes del presente estudio; Así, Jaén (2003) efectúa un estudio titulado "Predicción del rendimiento laboral a partir de indicadores de motivación, personalidad y percepción de factores psicosociales", en el cual establece como objeto fundamental del trabajo la comprobación del papel de las variables de personalidad, motivación y percepción de factores psicosociales en el rendimiento de los trabajadores, y evidenciar si aquellas variables son capaces de diferenciar entre los trabajadores de mejor rendimiento y los de peor rendimiento dentro del grupo de participantes. Los resultados que tiene la investigadora, en función de los diferentes análisis de regresión múltiple realizados (tanto utilizando como variable criterio el rendimiento global o general, como cada una de las seis dimensiones que conforman éste), conllevan a indicar que son las variables de Motivación y las Demandas Cognitivas percibidas en el entorno laboral, las que juegan un rol más importante. Respecto a la relación de las variables motivación rendimiento que integran una de las hipótesis planteadas para la investigación y en la cual se establecía la existencia de relación positiva y significativa entre las puntuaciones en motivación y la evaluación del rendimiento, es decir, que los individuos que obtuviesen puntuaciones más altas en motivación, tendrían un mejor rendimiento general en el trabajo, se llegó a la siguiente conclusión: se encontró una correlación significativa y en sentido positivo entre rendimiento y el nivel de Ejecución de 3 de los motivos evaluados: Reconocimiento Social, Autoestima y Autodesarrollo, aunque los valores de dichas relaciones sean muy bajos (0,14 en el mejor de los casos).

Otra investigación importante es la desarrollada por Navarro (2008), quien titula su tesis "Aportación al estudio de la satisfacción laboral de los profesionales técnicos del sector de la construcción: una aplicación cualitativa en la Comunidad Valenciana" trabajo que se plantea como objeto general el estudio empírico de la satisfacción laboral de los profesionales técnicos del sector de la construcción en la Comunidad Valenciana, desde la perspectiva de los propios investigados, a través de un enfoque cualitativo. Mediante la investigación se pretendió descubrir cómo viven, sienten y perciben su actividad laboral estos profesionales; analizar si se sienten satisfechos en la cotidianeidad de su trabajo; describir los aspectos generadores de mayor satisfacción laboral y los factores de insatisfacción laboral, así como profundizar en temas tan actuales como el estrés laboral y la conciliación entre su vida laboral y personal, entre otros. Pero no se pretende predecir ni universalizar extendiendo hacia el futuro o hacia otros casos los resultados; por el contrario, el interés se orienta a analizar casos concretos en su particularidad temporal y local y a partir de las expresiones de los propios investigados, de ahí la metodología cualitativa y su carácter exploratorio. En la investigación se concluye que, en general, los profesionales entrevistados están satisfechos con su trabajo en el sector de la construcción, disfrutan del mismo y describen globalmente su experiencia 
laboral en términos positivos. Los factores de satisfacción laboral más importantes son los relacionados con la naturaleza y el contenido del trabajo, es decir, con factores intrínsecos como la identidad, el interés, la variedad y la significatividad de las tareas realizadas, el reconocimiento obtenido y el reto cotidiano. Por el contrario, los factores de insatisfacción laboral más señalados son de carácter extrínseco $\mathrm{y}$ vinculado al contexto de trabajo.

La investigación efectuada y presentada en el presente artículo se desarrolló en base al problema evidenciado que se plantea mediante la siguiente interrogante: ¿De qué modo la motivación incide en el desempeño laboral de los trabajadores de las pequeñas empresas de la provincia de Manabí - Ecuador?. El objetivo principal que se estableció para la investigación desarrollada es: Determinar de qué manera la motivación incide en el desempeño laboral de los trabajadores de las pequeñas empresas de la provincia de Manabí - Ecuador.

En Manabí se percibe la existencia de muchas pequeñas empresas privadas en las cuales se considera que para conformar un equipo de trabajo eficiente, basta con contratar a quienes tengan el mejor currículum o cuenten con las habilidades requeridas para el cargo que realizarán posteriormente dentro de la entidad. La perspectiva asumida anteriormente por este tipo de empresas, puede convertirse en un criterio errado, debido a que no necesariamente es así.

Es fundamental hacer una medición constante del desempeño de los trabajadores una vez que son contratados, y medir el nivel de satisfacción laboral que tienen los mismos para establecer criterios técnicos asociados que permitan adoptar modelos conceptuales o decisiones administrativas estratégicas que sean pertinentes para el eficiente desarrollo del talento humano organizacional.

En lo teórico, los resultados derivados de la presente investigación pueden utilizarse en futuras investigaciones por cuanto se plantea conocimientos sobre los principales factores que están incidiendo en el desempeño laboral de los trabajadores de las pequeñas empresas privadas, mismos que pueden utilizarse como referentes por los investigadores académicos que efectúen estudios sobre temas relacionados. Paralelamente, la investigación aporta con fundamentos sobre la definición de motivación laboral considerando que la misma debe estar sustentada en principios y criterios socio económicos contemporáneos que permitan un eficiente y adecuado desempeño laboral de los trabajadores en sus distintas funciones dentro de una pequeña empresa privada, mismos que deben considerarse como un importante eje transversal en las estructuras organizativas adoptadas en las empresas.

En lo práctico, mediante las conclusiones que se establezcan en la investigación, las pequeñas empresas manabitas podrían identificar las debilidades de sus equipos de trabajo y así realizar planes de capacitación adecuados, que apunten a temas específicos y que consideren el entorno socio cultural de los trabajadores.

Este trabajo de investigación es de gran relevancia porque permitió obtener mediante las actividades del análisis bibliográfico, así como del estudio de campo, una información confiable y válida sobre las necesidades motivacionales de los trabajadores y elementos que determinan la sensación de bienestar que mantienen los mismos con relación a las funciones laborales efectuadas, las cuales sirvieron para establecer regularidades desde el campo empírico para efectos del planteo de un aporte práctico para las ciencias administrativas respecto de los procesos motivacionales de los trabajadores, su planeación e incidencias en el desempeño laboral. Es imprescindible que dentro de las organizaciones se evalúe constantemente la motivación que posee un trabajador como una herramienta para mejorar el desempeño laboral de los mismos, realizando ajustes o adaptaciones necesarias de acuerdo a los intereses que tenga la pequeña empresa. Los resultados expuestos y derivados del presente estudio, poseen como limitación principal el cambio emotivo que pueden tener los trabajadores encuestados en función de los factores de su entorno laboral que condicionan su desempeño, lo cuales son muy variables y evolucionan constantemente en relación a otros factores como la situación las políticas económicas del país, la tecnología, las leyes laborales, entre otras; mismas que no se consideran en la investigación efectuada. y las obras de Vía parque Rímac.

El término motivación se deriva de la latina "movere" cuya significa en español es "mover". La motivación es una característica que posee la psicología humana y que contribuye al nivel de compromiso que asume una persona ante una determinada situación (Chiavenato, 2000). La motivación es un proceso que ocasiona, activa, orienta, dinamiza y mantiene el comportamiento de los individuos hacia la realización de los objetivos esperados (López, 2005). La motivación, según Robbins (2004) representa la voluntad de ejercer altos niveles de esfuerzo hacia las metas organizacionales, condicionada por la capacidad del esfuerzo para satisfacer alguna necesidad individual. Palmero (2005), establece que 
la motivación es el conjunto de procesos de carácter integral e intangible que están relacionados con el propio sujeto y con el contexto, y que están implicados en la activación, dirección y persistencia de la conducta, tanto de manera consciente como inconsciente.

En el contexto laboral, según González y Olivares (1999) la motivación comprende tanto los procesos individuales que llevan a un trabajador a actuar y que se vinculan con su desempeño y satisfacción en la empresa, como los procesos organizacionales que influyen para que, tanto los motivos del trabajador como los de la empresa, vayan en la misma dirección. Las teorías generales sobre motivación se las clasifica en: 1) Teorías centradas en la persona; 2) Teorías centradas en el contexto; y, 3) Teorías centradas en la relación contexto - persona.

En las teorías centradas en la persona se ponen énfasis en el propio individuo motivado, aunque desde diferentes puntos de vista. Dentro de este grupo, a criterio del autor del artículo, las más relevantes son:

Alderfer (1969) plantea una revisión del modelo de Maslow y establece tres categorías de necesidades:

(E) Necesidades de existencia. Relacionadas con la supervivencia, (equivaldría a las fisiológicas de Maslow). (R) Necesidades de Relación interpersonal. Implicadas en los procesos de relaciones interpersonales. (C) Necesidades de Crecimiento o desarrollo personal. Implicadas en la propia valoración que el sujeto elabora sobre sí mismo

Alderfer propone una jerarquización de las necesidades menos estricta que la planteada por Maslow. Establece las siguientes relaciones entre los distintos tipos de necesidades: a) Es posible que estén activas dos o más necesidades simultáneamente, y se puede transitar de necesidades sin que sean satisfechas totalmente unas para pasar a las siguientes b) Cuanto menos está satisfecha una necesidad superior, más se desea la satisfacción de una necesidad inferior. c) Al satisfacer una necesidad, más se desea la satisfacción de necesidades de orden superior.

Deci y Ryan (1985) señalan que la motivación intrínseca es aquélla que nace del interior de la persona con el fin de satisfacer sus deseos de autorrealización y crecimiento personal. La motivación intrínseca no nace con el objetivo de obtener resultados, sino que nace del placer que se obtiene al realizar una tarea, es decir, al proceso de realización en sí; la motivación intrínseca posee elementos que la estructuran:

Necesidad de competencia llevas a las personas a buscar y conseguir retos que son óptimos para sus capacidades; la adquisición de competencia resulta de la interacción con estímulos que suponen desafíos.

Necesidad de autodeterminación las personas buscan tener el control de sus propias acciones, siendo este deseo el núcleo mismo de la conducta motivada intrínsecamente.

Las teorías centradas en el contexto explican el refuerzo como la inducción de una pauta de comportamiento determinada a través de la presentación reiterada de consecuencias positivas o negativas tras la ejecución de conductas que se asemejen o difieran de la pauta que se quiere establecer. En esta teoría, no se da especial importancia a los factores emocionales o fisiológicos, dentro de las cuales se destaca: Locke y Latham (1990) reconocen un papel motivacional central a las intenciones de los sujetos al realizar una tarea. Son los objetivos o metas que los sujetos persiguen con la realización de la tarea los que determinarán el nivel de esfuerzo que emplearán en su ejecución. El modelo trata de explicar los efectos de esos objetivos sobre el rendimiento.

Los objetivos son los que determinan la dirección del comportamiento del sujeto y contribuyen a la función energitizante del esfuerzo. Los cambios en los valores de los incentivos pueden sólo afectar a su comportamiento en la medida en que vayan asociados a los cambios de objetivos. La satisfacción de los individuos con su rendimiento estará en función del grado de consecución de los objetivos permitido por ese rendimiento. La teoría del establecimiento de metas $\mathrm{u}$ objetivos supone que las intenciones de trabajar para conseguir un determinado objetivo es la primera fuerza motivadora del esfuerzo laboral y determina el esfuerzo desarrollado para la realización de tareas. La investigación a partir del modelo ha permitido formular conclusiones relevantes para la motivación del comportamiento en el contexto organizacional.

McClelland (1985) sostuvo que todos los individuos poseen necesidades que los motivan en su accionar o comportamiento, a saber:

Necesidad de logro deseo que tienen las personas de hacer cada vez mejor las cosas; es decir, el deseo de una superación personal a través del éxito en sus realizaciones y de la evitación del fracaso. Necesidad de afiliación necesidad de establecer, mantener o renovar relaciones afectivas o de amistad con otras personas. Necesidad de poder necesidad de 
tener influencia sobre los demás, posibilidad de ejercer un control sobre ellos y deseo de dominar.

Finalmente las teorías centradas en la relación entre el contexto y la persona, consideran que el individuo no es ajeno al ambiente que lo rodea, tampoco al modo en que la organización y la cultura interfieren en su comportamiento y en la manera en que este individuo interpreta esa realidad. Bajo ese enfoque se estructuran las teorías expuestas a continuación: Vroom (1979) propone que la motivación es producto de la valencia o el valor que el individuo pone en los posibles resultados de sus acciones y la expectativa de que sus metas se cumplan. La importancia de esta teoría es la insistencia que hace en la individualidad y la variabilidad de las fuerzas motivadoras. El fundamento de este método se basa en cuatro supuestos: 1. El comportamiento depende de la combinación de las fuerzas de las personas y del medio que lo rodea. 2. Las personas toman las decisiones conscientes sobre su comportamiento. 3. Las personas tienen distintas necesidades, deseos y metas. 4. Las personas escogen entre distintas opciones de comportamientos.

Se basa en que el esfuerzo para obtener un alto desempeño, está en dependencia de la posibilidad de lograr el desempeño y que una vez alcanzado sea recompensado de tal manera que el esfuerzo realizado haya valido la pena.

La teoría de la Equidad intenta explicar la satisfacción relacional en términos de percepciones de tarifas / distribuciones injustas de recursos dentro de las relaciones interpersonales. Considerada una de las teorías de la justicia por cuanto en la misma se afirma que los empleados buscan mantener la equidad entre los insu- mos que traen a un puesto de trabajo y los resultados que reciben de ella contra las entradas percibidas y los resultados de los demás (Adams, 1965).

La creencia es el trato justo del valor de personas lo que les provoca motivación para mantener la imparcialidad que se mantiene dentro de las relaciones de la organización y con sus compañeros de trabajo. La estructura de la equidad en el lugar de trabajo está basada en la proporción de insumos a los resultados. Insumos son las contribuciones hechas por el empleado de la organización.

Por lo tanto un individuo tendrá en cuenta que se trató bastante si percibe la proporción de sus aportaciones a sus resultados equivalentes a los que le rodean y sería aceptable para un colega más alto a recibir una indemnización mayor, puesto que el valor de su experiencia (y entrada) es mayor que el propio.

\section{MATERIAL Y MÉTODOS}

La investigación realizada es de tipo Cuali - Cuantitativa, y No Experimental; además la investigación tuvo un nivel descriptivo y exploratorio, por cuanto se analizan y explican los niveles de motivación en el desempeño laboral de los trabajadores de las pequeñas empresas privadas de la provincia Manabí y algunas de sus posibles causas. Igualmente la investigación realizada fue de tipo bibliográfico, por cuanto se acudió a la revisión de textos especializados en el tema para estructurar el marco teórico en el cual se sustenta el estudio. Se utilizó la estadística como técnica puesto que mediante una encuesta se recopiló información cualitativamente que se ponderó, para posteriormente en base a la misma establecer la relación que se presenta entre la motivación y el desempeño de los trabajadores.
La investigación se efectuó en las pequeñas empresas de la provincia de Manabí, mismas que poseen características específicas señaladas en la norma legal societaria vigente en el Ecuador. La población está constituida por los trabajadores de la pequeñas empresas de la provincia de Manabí. El tamaño de la muestra de los trabajadores analizados fue de 200. Para la selección de la muestra se empleó el muestreo intencional considerando únicamente a los trabajadores que tienen funciones directamente vinculadas con el área de producción y que poseen 2 o más años en relación de dependencia con la empresa.

\section{RESULTADOS}

A continuación se exponen los resultados y análisis de las encuestas que se aplicaron a los trabajadores de las pequeñas empresas, mediante las cuales se establece el nivel de satisfacción que poseen, los elementos motivacionales más importantes para ellos, y la relación de la motivación que mantienen con el desempeño dentro de sus funciones operativas.

En la Tabla 1 se puede apreciar que aproximadamente un $70 \%$ de los trabajadores se encuentran satisfechos o muy satisfechos dentro de su empresa, y el restante $30 \%$ demuestra una insatisfacción dentro de las funciones que desempeña; la información obtenida se la complementó con los datos que se presentan en la siguiente tabla, respecto de la insatisfacción y satisfacción de los trabajadores en función de los años de permanencia en la empresa y el nivel de educación escolar que poseen al momento de la encuesta (E.B = Educación Básica; E.M = Educación Media; E.S = Educación Superior):

Mientras en la Tabla 2 muestra que el nivel de satisfacción de los trabaja- 
dores se relaciona de manera inversamente proporcional con el nivel de estudios que poseen, lo cual implica que entre más elevado sea el nivel de estu-

Tabla 1.

Nivel de satisfacción en el trabajo

Fuente: Encuesta aplicada a los trabajadores / Elaboración de autor

\begin{tabular}{lccc}
\multicolumn{1}{c}{ Alternativas } & Cantidad & Frecuencia \% & Acumulado \% \\
Muy satisfecho (4) & 52 & $26 \%$ & $26 \%$ \\
\hline Satisfecho (3) & 87 & $43,5 \%$ & $69,5 \%$ \\
\hline Insatisfecho (2) & 35 & $17,5 \%$ & $87 \%$ \\
\hline Muy insatisfecho (1) & 26 & $13 \%$ & $100 \%$ \\
Total & $\mathbf{2 0 0}$ & $\mathbf{1 0 0 \%}$ &
\end{tabular}

Tabla 2.

Nivel de satisfacción en el trabajo relacionada con el nivel de estudios Fuente: Encuesta aplicada a los trabajadores / Elaboración de autor

\begin{tabular}{lcccc}
\multicolumn{1}{c}{ Alternativas } & Cantidad & E.B & E.M & E.S \\
Muy satisfecho (4) & 52 & 39 & 12 & 1 \\
\hline Satisfecho (3) & 87 & 45 & 32 & 10 \\
\hline Insatisfecho (2) & 35 & 3 & 12 & 20 \\
\hline Muy insatisfecho (1) & 26 & 0 & 3 & 23 \\
Total & $\mathbf{2 0 0}$ & $\mathbf{8 7}$ & $\mathbf{5 9}$ & $\mathbf{5 4}$
\end{tabular}

Tabla 3.

Nivel de satisfacción en el trabajo relacionada con los años de trabajo Fuente: Encuesta aplicada a los trabajadores / Elaboración de autor

\begin{tabular}{lcccc}
\multicolumn{1}{c}{ Alternativas } & Cantidad & $\mathbf{2 - 4}$ años & $\mathbf{4 - 6}$ años & $\mathbf{6}$ o más años \\
Muy satisfecho (4) & 52 & 27 & 21 & 4 \\
\hline Satisfecho (3) & 87 & 41 & 33 & 13 \\
\hline Insatisfecho (2) & 35 & 3 & 9 & 23 \\
\hline Muy insatisfecho (1) & 26 & 1 & 8 & 17 \\
Total & $\mathbf{2 0 0}$ & $\mathbf{7 2}$ & $\mathbf{7 1}$ & $\mathbf{5 7}$
\end{tabular}

Tabla 4.

Nivel de motivación recibida por los trabajadores

Fuente: Encuesta aplicada a los trabajadores / Elaboración de autor

\begin{tabular}{lccc}
\multicolumn{1}{c}{ Alternativas } & Cantidad & Frecuencia \% & Acumulado \% \\
Muy de acuerdo (4) & 57 & $28,5 \%$ & $28,5 \%$ \\
\hline De acuerdo (3) & 79 & $39,5 \%$ & $68 \%$ \\
\hline En desacuerdo (2) & 42 & $21 \%$ & $89 \%$ \\
\hline Muy en desacuerdo (1) & 22 & $11 \%$ & $100 \%$ \\
Total & $\mathbf{2 0 0}$ & $\mathbf{1 0 0 \%}$ &
\end{tabular}

que tienen los trabajadores se relaciona de una manera inversamente proporcional con el tiempo de trabajo que tienen dentro de la empresa, lo cual implica que entre más años de trabajo en la empresa, el trabajador evidencia menor nivel de satisfacción en sus labores, y viceversa. En base a lo expuesto, se puede inferir que los trabajadores con mayor nivel educativo y mayor cantidad de años dentro de sus labores, representan un alto riesgo de deserción para la empresa debido a la disminución periódica de la motivación que mantienen para el desarrollo cotidiano de las funciones que poseen. Es importante acotar que durante la investigación de campo se obtuvo también información que demuestra que los trabajadores que tienen mayor nivel educativo y mayor tiempo dentro de las empresas, se motivan mucho más por el trato que reciben de sus jefes inmediatos, reconocimiento de sus labores y ambiente laboral, que por la remuneración que reciben como retribución al trabajo efectuado; situación que se presenta con menor intensidad en los trabajadores con menos nivel educativo y menor cantidad de años de labores dentro de las empresas analizadas, quienes evidenciaron mayor motivación por la parte remunerativa.

En la Tabla 4, un $32 \%$ de los trabajadores que manifiestan tener un desacuerdo con la gestión de motivación que reciben dentro de la empresa para el desarrollo de las funciones y tareas asignadas, situación que puede variar en función de variables externas que afectan las percepciones emocionales de los trabajadores, entre las cuales se destacan la estabilidad y seguridad familiar.

En la Tabla 5, la mayoría de los trabajadores, esto es el $73 \%$ de los mismos, opinan que el reconocimiento 
efectivizado por los jefes tiene un efecto directo sobre el nivel de desempeño que tienen dentro de la empresa. Es importante acotar que estos reconocimientos deben ser aplicados de manera reglamentaria considerando parámetros de eficiencia, eficacia y productividad que deben ser socializados con todos los integrantes de la organización, lo cual evitará percepciones en los trabajadores de preferencias e injusticias que se convertirían en elementos potencialmente desmotivadores.

Como se aprecia en la Tabla 6, el $75,5 \%$ de los empleados considera que el nivel educativo escolarizado que poseen, contribuye poco o nada con el mejoramiento del desempeño laboral que tienen dentro del área productiva operativa de la empresa.

En tanto, en la Tabla 7, se puede apreciar de manera objetiva que el entorno laboral es un factor motivacional importante para el desempeño de los trabajadores de la pequeña empresa, lo cual se corrobora con la opinión del $81,5 \%$ de los trabajadores encuestados.

Finalmente en la Tabla 8, se estableció que la mayoría de los trabajadores de las pequeñas empresas, representado por el $75 \%$, no están de acuerdo con la remuneración que perciben por concepto de las labores que efectúan.

\section{DISCUSIÓN}

Los trabajadores analizados mantienen niveles de motivación diferentes, los mismos que están afectados por el nivel educativo que poseen y la cantidad de años que tienen dentro de la organización. La motivación de los trabajadores para su desempeño laboral mantiene una relación inversa proporcional con el nivel educativo y cantidad de años de labores que poseen los mismos. Los trabajadores que poseen mayor nivel educativo y mayor cantidad de años de labores dentro de las empresas,

valoran mucho más, como elemento motivacional de su desempeño, el reconocimiento de sus labores, el trato que le brindan sus jefes y el entorno la-

\section{Tabla 5.}

El reconocimiento de los jefes como factor motivacional

Fuente: Encuesta aplicada a los trabajadores / Elaboración de autor

\begin{tabular}{lccc}
\multicolumn{1}{c}{ Alternativas } & Cantidad & Frecuencia \% & Acumulado \% \\
Muy de acuerdo (4) & 87 & $43,5 \%$ & $43,5 \%$ \\
\hline De acuerdo (3) & 59 & $29,5 \%$ & $73 \%$ \\
\hline En desacuerdo (2) & 33 & $16,5 \%$ & $89,5 \%$ \\
\hline Muy en desacuerdo (1) & 21 & $10,5 \%$ & $100 \%$ \\
Total & $\mathbf{2 0 0}$ & $\mathbf{1 0 0 \%}$ &
\end{tabular}

Tabla 6.

El nivel educativo escolarizado como factor motivacional

Fuente: Encuesta aplicada a los trabajadores / Elaboración de autor

\begin{tabular}{lccc}
\multicolumn{1}{c}{ Alternativas } & Cantidad & Frecuencia \% & Acumulado \% \\
Mucho (4) & 12 & $6 \%$ & $6 \%$ \\
\hline Lo suficiente (3) & 37 & $18,5 \%$ & $24,5 \%$ \\
\hline Poco (2) & 96 & $48 \%$ & $72,5 \%$ \\
\hline Nada (1) & 55 & $27,5 \%$ & $100 \%$ \\
Total & $\mathbf{2 0 0}$ & $\mathbf{1 0 0 \%}$ &
\end{tabular}

\section{Tabla 7.}

El entorno laboral como factor motivacional

Fuente: Encuesta aplicada a los trabajadores / Elaboración de autor

\begin{tabular}{lccc}
\multicolumn{1}{c}{ Alternativas } & Cantidad & Frecuencia \% & Acumulado \% \\
Muy de acuerdo (4) & 105 & $52,5 \%$ & $52,5 \%$ \\
\hline De acuerdo (3) & 58 & $29 \%$ & $81,5 \%$ \\
\hline En desacuerdo (2) & 25 & $12,5 \%$ & $94 \%$ \\
\hline Muy en desacuerdo (1) & 12 & $6 \%$ & $100 \%$ \\
Total & $\mathbf{2 0 0}$ & $\mathbf{1 0 0 \%}$ &
\end{tabular}

Tabla 8.

La remuneración como factor motivacional

Fuente: Encuesta aplicada a los trabajadores / Elaboración de autor

\begin{tabular}{lccc}
\multicolumn{1}{c}{ Alternativas } & Cantidad & Frecuencia $\%$ & Acumulado \% \\
Totalmente (4) & 26 & $13 \%$ & $13 \%$ \\
\hline Mucho (3) & 24 & $12 \%$ & $25 \%$ \\
\hline Parcialmente (2) & 145 & $72,5 \%$ & $87,5 \%$ \\
\hline Nada (1) & 5 & $2,5 \%$ & $100 \%$ \\
Total & $\mathbf{2 0 0}$ & $\mathbf{1 0 0 \%}$ &
\end{tabular}


boral en el cual se desempeñan, que la parte económica remunerativa que la empresa le entrega como retribución.

La motivación que tiene un trabajador para el desarrollo de sus labores sufre variaciones en función de aspectos exógenos que afectan la inteligencia emocional que mantienen los mismos, entre los cuales se destaca la tranquilidad y seguridad de los miembros de su familia.

Una utilización inadecuada de estrategias motivacionales dirigida a los trabajadores, incidirá en el incremento de los niveles de desmotivación de los mismos y por ende en el desempeño laboral que tienen dentro de las organizaciones.

\section{REFERENCIAS BIBLIOGRÁFICAS}

Adams, J. (1965). Inequity in social Exchange. Advances in Experimental Social Psychology, 2, pp. 267-299

Alderfer, C. (1969). An empirical test of new theory of human needs. Organizational Behavior and Human Performance, 4(1), pp. 142-175.
Chiavenato, I. (2000). Administración de recursos humanos. (5ta ed.). Bogotá, Colombia: Mc. Graw Hill.

Deci, E. \& Ryan, R. (1985). Intrinsic motivation and self-determination in human behavior. New York, EE.UU.: Plemuim.

González, M. \& Olivares, S. (1999). Comportamiento organizacional: Un enfoque latinoamericano. México D.F., México: CECSA.

Jaén, M. (2003). Predicción del rendimiento laboral a partir de indicadores de motivación, personalidad y percepción de factores psicosociales. (Tesis doctoral). Universidad Complutense de Madrid, Madrid, España.

Locke, E. \& Latham, G. (1990). Work motivation and satisfaction: Light at the end of the tunnel. American Psychological Society, 1(4), pp. 240246.

López, A. (2005). La Motivación. México D.F., México: Mc Graw-Hill Inte- ramericana.

Maslow, A. H. (1970). Motivación y personalidad. (3ra ed.). Madrid, España: Ediciones Díaz de Santos S.A.

McClelland, D. (1985). Human Motivation. New York, EE.UU.: Scott, Foresman and Co.

Navarro, E. (2008). Aportación al estudio de la satisfacción laboral de los profesionales técnicos del sector de la construcción. (Tesis doctoral). Universidad Politécnica de Valencia, Valencia, España.

Palmero, F. (2005). Motivación y emoción. Madrid, España: McGraw-Hill Interamericana.

Robbins, S. P. (2004). Comportamiento organizacional. (10ma ed.). México D.F., México: Pearson Educación.

Vroom, V. (1979). Motivación y alta dirección. México D.F., México: Trillas. 